\title{
Lipid Metabolism in Biliary Tract Cancer: A New Therapeutic Target?
}

\author{
Daniel M. Kerekes, MD, and Sajid A. Khan, MD, FACS, FSSO \\ Division of Surgical Oncology, Department of Surgery, Yale School of Medicine, New Haven, CT
}

A recent study by Ueno et al. ${ }^{1}$ aims to cast light on the issue of gemcitabine resistance in the treatment of biliary tract cancers (BTC). The authors begin by demonstrating a key metabolic distinction between gemcitabine-resistant (GR) and gemcitabine-sensitive (GS) BTC cells. They show that acyl-coenzyme A (cholesterol acyltransferase-1 [ACAT-1]), an endoplasmic reticulum enzyme that converts free cholesterol to cholesteryl ester (CE) for storage, is increased in GR human gallbladder cancer cells relative to their GS parent cells. The authors go on to show with in vitro experiments that either knockdown of ACAT-1 or inhibition with avasimibe in GR tumor cells improves sensitivity to gemcitabine via endoplasmic reticulum stress, thus suggesting ACAT-1 as a possible novel therapeutic target in GR BTC. Finally, they bolster their findings with evidence that ACAT-1 expression level is prognostic of survival for gemcitabine-treated patients with intrahepatic cholangiocarcinoma (IHCCA).

Although surgical resection offers the best chance at long-term survival for BTC patients, fewer than one third of patients qualify for curative resection at the time of presentation. $^{2}$ Furthermore, those who undergo resection experience high recurrence rates and often die of metastatic disease. ${ }^{3,4}$ As such, chemotherapy plays an important role in the treatment of most BTC patients. However, current options have limited efficacy. Gemcitabine-based chemotherapy represents the standard of care for unresectable disease, yet the survival benefit for these regimens

(C) Society of Surgical Oncology 2022

First Received: 23 November 2021

Accepted: 18 January 2022;

Published Online: 3 March 2022

S. A. Khan, MD, FACS, FSSO

e-mail: Sajid.Khan@yale.edu is only about 3 to 5 months. Intrinsic and acquired resistance to the agents is high, and disease progression is the rule rather than the exception. ${ }^{2,5,6}$ A more effective gemcitabine-based regimen for treatment of BTC would therefore represent a significant contribution to the field.

Recent years have seen an emerging role for cancer metabolomics in the management of solid tumor malignancies. $^{7,8}$ Some previously overshadowed metabolic pathways are currently recognized as playing a significant role in tumor growth and response to therapy, such as the cholesterol storage pathway investigated in this study by Ueno et al. ${ }^{1}$ and by others in malignancies of the pancreas, breast and prostate. ${ }^{9-11}$

We commend the authors for demonstrating a major metabolic distinction in lipid metabolism between GR and GS BTC cells. The observed association between increased ACAT-1 and acquired gemcitabine resistance is further underscored by their finding that BTC cell lines with higher baseline ACAT-1 expression have higher intrinsic resistance to gemcitabine in a concentration-dependent fashion. The authors show that targeting this metabolic pathway helps to revert GR cells to the GS phenotype. Importantly, they demonstrate this using a well-tolerated drug whose safety in humans has already been established across a 10-fold range of doses. ${ }^{12}$ This study design paves the way for translation into animal studies with potential for clinical application.

Although these results are promising, they are demonstrated in only one cell line, and BTC is an unusually heterogenous disease, both in cellular origin and in genomic variability. ${ }^{2}$ Comparable results must be demonstrated in additional BTC cell lines. Indeed, previous work on IHCCA has shown that doxycycline also facilitates gemcitabine re-sensitization. ${ }^{13}$ Future studies investigating possible synergy between doxycycline and avasimibe in GR IHCCA could be fruitful. 
Finally, although a clear relationship between increased ACAT-1 and gemcitabine resistance has been demonstrated, the mechanism behind the association needs to be explored further. As mentioned by the authors, CD8+ T lymphocytes, the MAPK pathway, and the PI3k/Akt pathway all have been previously implicated as mediators of the effect that ACAT-1 inhibition has on cancer growth suppression. ${ }^{9,14,15}$ In vivo studies testing these pathways may provide supporting evidence for a model of the CEgemcitabine relationship in BTC.

Current systemic therapy options for BTC are characterized by patterns of resistance and low efficacy. However, recent advances in metabolomics currently are providing insights into aspects of the altered metabolism of these cancers that may prove susceptible to disruption, offering hope for novel treatment strategies.

\section{REFERENCES}

1. Ueno G, Iwagami Y, Kobayashi S, Mitsufuji S, Yamada D, Tomimaru Y. ACAT-1-regulated cholesteryl ester accumulation modulates gemcitabine resistance in biliary tract cancer. Ann Surg Oncol. 2022. https://doi.org/10.1245/s10434-021-11152-1

2. Banales JM, Marin JJG, Lamarca A, et al. Cholangiocarcinoma 2020: the next horizon in mechanisms and management. Nat Rev Gastroenterol Hepatol. 2020;17:557-88. https://doi.org/10.1038/ s41575-020-0310-z.

3. Mavros MN, Economopoulos KP, Alexiou VG, Pawlik TM. Treatment and prognosis for patients with intrahepatic cholangiocarcinoma: systematic review and meta-analysis. JAMA Surg. 2014;149:565-74. https://doi.org/10.1001/jamasurg.2013.5137.

4. Jang JY, Kim SW, Park DJ, et al. Actual long-term outcome of extrahepatic bile duct cancer after surgical resection. Ann Surg. 2005;241:77-84. https://doi.org/10.1097/01.sla.0000150166.947 32.88 .

5. Valle J, Wasan H, Palmer DH, et al. Cisplatin plus gemcitabine versus gemcitabine for biliary tract cancer. $N$ Engl J Med. 2010;362:1273-81. https://doi.org/10.1056/NEJMoa0908721.

6. Okusaka T, Nakachi K, Fukutomi A, et al. Gemcitabine alone or in combination with cisplatin in patients with biliary tract cancer: a comparative multicentre study in Japan. $\mathrm{Br} J$ Cancer. 2010;103:469-74. https://doi.org/10.1038/sj.bjc.6605779.

7. Cai Y, Rattray NJW, Zhang Q, et al. Tumor tissue-specific biomarkers of colorectal cancer by anatomic location and stage. Metabolites. 2020;10:257. https://doi.org/10.3390/metab o10060257.

8. Vander Heiden MG, DeBerardinis RJ. Understanding the intersections between metabolism and cancer biology. Cell. 2017;168:657-9. https://doi.org/10.1016/j.cell.2016.12.039.

9. Li J, Qu X, Tian J, Zhang JT, Cheng JX. Cholesterol esterification inhibition and gemcitabine synergistically suppress pancreatic ductal adenocarcinoma proliferation. PloS One. 2018;13:e0193318. https://doi.org/10.1371/journal.pone. 0193318.

10. Poirot M, Silvente-Poirot S, Weichselbaum RR. Cholesterol metabolism and resistance to tamoxifen. Curr Opin Pharmacol. 2012;12:683-9. https://doi.org/10.1016/j.coph.2012.09.007.

11. Yue S, Li J, Lee SY, et al. Cholesteryl ester accumulation induced by PTEN loss and PI3K/AKT activation underlies human prostate cancer aggressiveness. Cell Metab. 2014;19:393-406. h ttps://doi.org/10.1016/j.cmet.2014.01.019.

12. Insull W, Koren M, Davignon J, et al. Efficacy and short-term safety of a new ACAT inhibitor, avasimibe, on lipids, lipoproteins, and apolipoproteins, in patients with combined hyperlipidemia. Atherosclerosis. 2001;157:137-44. https://doi.or g/10.1016/s0021-9150(00)00615-8.

13. Varamo C, Peraldo-Neia C, Ostano P, et al. Establishment and characterization of a new intrahepatic cholangiocarcinoma cell line resistant to gemcitabine. Cancers Basel. 2019;11:519. http s://doi.org/10.3390/cancers11040519.

14. Li M, Yang Y, Wei J, et al. Enhanced chemo-immunotherapy against melanoma by inhibition of cholesterol esterification in CD8+ T cells. Nanomedicine. 2018;14:2541-50. https://doi.org/ 10.1016/j.nano.2018.08.008.

15. Bandyopadhyay S, Li J, Traer E, et al. Cholesterol esterification inhibition and imatinib treatment synergistically inhibit growth of BCR-ABL mutation-independent resistant chronic myelogenous leukemia. Plos One. 2017;12:e0179558. https://doi.org/10.1371/ journal.pone.0179558.

Publisher's Note Springer Nature remains neutral with regard to jurisdictional claims in published maps and institutional affiliations. 